\title{
Strength of Temporal White Matter Pathways Predicts Semantic Learning
}

\author{
Pablo Ripollés, ${ }^{1,2,3}$ @Davina Biel, ${ }^{1,4}$ @Claudia Peñaloza, ${ }^{1,5}$ Jörn Kaufmann, ${ }^{6}$ Josep Marco-Pallarés, ${ }^{1,2}$ \\ Toemme Noesselt, ${ }^{7,8 *}$ and ${ }^{\circledR}$ Antoni Rodríguez-Fornells ${ }^{1,2,9 *}$ \\ ${ }^{1}$ Cognition and Brain Plasticity Group, Bellvitge Biomedical Research Institute, and ${ }^{2}$ Department of Cognition, Development and Education Psychology, \\ Campus Bellvitge, University of Barcelona, L'Hospitalet de Llobregat, 08097 Barcelona, Spain, ${ }^{3}$ Poeppel Laboratory, Department of Psychology, New York \\ University, New York, New York 10003, ${ }^{4}$ Institute of Psychology I, University of Lübeck, 23562 Lübeck, Germany, ${ }^{5}$ Aphasia Research Laboratory, \\ Department of Speech, Language, and Hearing Sciences, Boston University, Boston, Massachusetts 02215, ${ }^{6}$ Department of Neurology, Otto-von-Guericke- \\ University Magdeburg, 39120 Magdeburg, Germany, 'Department of Biological Psychology, Otto-von-Guericke-University Magdeburg, 39106 Magdeburg, \\ Germany, ${ }^{8}$ Center for Behavioral Brain Sciences, D-39106 Magdeburg, Germany, and ${ }^{9}$ Catalan Institution for Research and Advanced Studies, Institució \\ Catalana de Recerca i Estudis Avançats, 08010 Barcelona, Spain
}

Learning the associations between words and meanings is a fundamental human ability. Although the language network is cortically well defined, the role of the white matter pathways supporting novel word-to-meaning mappings remains unclear. Here, by using contextual and cross-situational word learning, we tested whether learning the meaning of a new word is related to the integrity of the languagerelated white matter pathways in 40 adults ( 18 women). The arcuate, uncinate, inferior-fronto-occipital and inferior-longitudinal fasciculi were virtually dissected using manual and automatic deterministic fiber tracking. Critically, the automatic method allowed assessing the white matter microstructure along the tract. Results demonstrate that the microstructural properties of the left inferior-longitudinal fasciculus predict contextual learning, whereas the left uncinate was associated with cross-situational learning. In addition, we identified regions of special importance within these pathways: the posterior middle temporal gyrus, thought to serve as a lexical interface and specifically related to contextual learning; the anterior temporal lobe, known to be an amodal hub for semantic processing and related to cross-situational learning; and the white matter near the hippocampus, a structure fundamental for the initial stages of new-word learning and, remarkably, related to both types of word learning. No significant associations were found for the inferior-fronto-occipital fasciculus or the arcuate. While previous results suggest that learning new phonological word forms is mediated by the arcuate fasciculus, these findings show that the temporal pathways are the crucial neural substrate supporting one of the most striking human abilities: our capacity to identify correct associations between words and meanings under referential indeterminacy.

Key words: cross-situational learning; meaning; semantic; temporal pathways; tractography; word learning

\section{Significance Statement}

The language-processing network is cortically (i.e., gray matter) well defined. However, the role of the white matter pathways that support novel word learning within this network remains unclear. In this work, we dissected language-related (arcuate, uncinate, inferior-fronto-occipital, and inferior-longitudinal) fasciculi using manual and automatic tracking. We found the left inferiorlongitudinal fasciculus to be predictive of word-learning success in two word-to-meaning tasks: contextual and cross-situational learning paradigms. The left uncinate was predictive of cross-situational word learning. No significant correlations were found for the arcuate or the inferior-fronto-occipital fasciculus. While previous results showed that learning new phonological word forms is supported by the arcuate fasciculus, these findings demonstrate that learning new word-to-meaning associations is mainly dependent on temporal white matter pathways.

\section{Introduction}

Learning new words and meanings is a fundamental aspect of first and second language acquisition, representing a continuous challenge for humans throughout their life span. However, there is a current lack of understanding regarding the brain networks supporting word-to-meaning mappings. Establishing a link be- 
tween a new word and a set of representations (Allport, 1985) could be governed by different mechanisms with different task requirements: from simple associative ones in which a new label is fast glued to an external referent, to more subtle and continuous learning processes, in which repeated encounters with a new word in different contexts allows for a gradual inference of its meaning (Nation, 2001).

In addition to task requirements, subject-specific differences in performance can be linked to differences in neuroanatomy. The microstructural properties of white matter (WM) pathways - which constrain the flow of information across brain areas-convey reliable information about the role of these pathways and their connected regions in supporting particular cognitive processes (Behrens and Johansen-Berg, 2005; Kanai and Rees, 2011). With the aim of identifying - by performing in vivo dissections-the WM pathways supporting word-to-meaning mappings, we collected diffusion-weighted MRI (DW-MRI) data on 40 healthy adults who completed two semantic learning tasks that relied on different processes: (1) a contextual learning task (CTXL; Mestres-Missé et al., 2008; Ripollés et al., 2014) and (2) a crosssituational learning paradigm (XSL; Yu and Smith, 2007). During CTXL, possible meaning candidates that correspond with the contextual information available in a learning instance (i.e., sentences) are gradually narrowed down until reaching a correct word-to-meaning mapping through inferential processes (van Daalen-Kapteijns et al., 2001). In contrast, XSL allows correct identification of word-to-meaning mappings through the computation of cross-situational statistics by tracking the frequency of co-occurrence between words and referents (Smith et al., 2014; but for alternative hypothesis testing accounts of XSL, see Medina et al., 2011; Trueswell et al., 2013). While XSL neuroimaging studies are lacking, previous research using functional MRI (fMRI), shows that, in CTXL, learning word-to-meaning mappings is mediated by ventral inferior frontal (BA 47) and posterior middle temporal regions (Mestres-Missé et al., 2008, 2009; Ripollés et al., 2014). Although the inferior-fronto-occipital (IFOF) and the inferior-longitudinal fasciculi (ILF) are thought to mediate a direct connection between occipitofrontal and occipitotemporal areas, respectively (Catani et al., 2003; Forkel et al., 2014), research shows that information could flow from the posterior middle temporal gyrus (pMTG) - a hub in which several major WM pathways converge- to the ventral inferior frontal gyrus (IFG) through two routes: directly via the IFOF (Turken and Dronkers, 2011) or using a two-step alternative pathway comprising the ILF [ending in the anterior temporal lobe (ATL)] and the uncinate fasciculus (UF; connecting the anterior temporal lobe to the IFG; Vigneau et al., 2006). However, a discrepancy exists about which of these pathways support general languagerelated processes, let alone word-to-meaning mappings. While several findings suggest that the ILF (Saur et al., 2008; Shinoura et al., 2010; Wong et al., 2011) and the UF do have a role in semantic

Profesorado Universitario program Grant AP2010-4179 awarded to P.R.], Generalitat de Catalunya (Grup Consolidat Generalitat de (atalunya, Grant 2014SGR1413 awarded to A.R.F.), and the Deutsche Forschungsgemeinschaft (Grant SFB779/TPA15 to T.N.). We thank T. Pohl, D. Scheermann, and K. Moehring for their help scanning the participants, J. Sierpowska and L. Vaquero for their help dissecting the tracts, and J.R. King for his assistance with the cross-validation analyses.

*T.N. and A.R.-F. shared senior authorship.

The authors declare no competing financial interests.

Correspondence should be addressed to Antoni Rodríguez-Fornells, Cognition and Brain Plasticity Group [Bellvitge Biomedical Research Institute-IDIBELL], L'Hospitalet de Llobregat, Barcelona, 08097 Spain. E-mail: arfornells@gmail.com.

DOI:10.1523/JNEUROSCI.1720-17.2017

Copyright $\odot 2017$ the authors $\quad 0270-6474 / 17 / 3711102-13 \$ 15.00 / 0$ processing (Papagno et al., 2011; Dick and Tremblay, 2012; Harvey et al., 2013), other research tends to favor the IFOF as the main semantic pathway (Duffau et al., 2005, 2009; Mandonnet et al., 2007).

To characterize the potential role of the IFOF, ILF, and UF in different types of semantic learning [the arcuate fasciculus (AF) was also dissected as a control, given its role in learning new phonological word-forms; López-Barroso et al., 2013], we applied both manual dissections (in which an average diffusivity value for a whole pathway is taken) and an automatic method, which allowed for the computation independent values along a particular tract. The latter method can provide information about the parts of the tract instrumental for semantic learning, as axons do not always run along a whole tract and can enter or exit at different anatomical positions within a pathway (Yeatman et al., 2012).

\section{Materials and Methods}

Participants. Forty German speakers (mean \pm SD age, $24.78 \pm 4.7$ years; 18 women; same as in Ripollés et al., 2014) were recruited from the student population at Otto-von-Guericke-University (Magdeburg, Germany). All participants were right handed, gave their informed written consent, and were paid or received course credits for their participation in accordance with local ethics. Stimuli were presented using the Psychophysics Toolbox 3.09 (Brainard, 1997) and Matlab version R2011b (7.13.0.564, 32 bit).

Contextual learning paradigm. For CTXL, we used the same paradigm as previous research (Ripollés et al., 2014, 2016). In this paradigm, participants were required to read 80 duplets of sentences that always ended in a new word. These words respected the phonotactic rules of German and were artificially created by changing one or two letters of an existing word. The nouns to be learned were selected from the CELEX database (mean frequency, 46.5 per million; SD, 22.85). Participants can derive and learn the meaning of new words using the semantic context provided by the duplets of sentences as they are built with an increasing degree of contextual constraint (Mestres-Missé et al., 2008, 2009, 2010; Ripollés et al., 2014). The mean cloze probability (the proportion of people who complete a particular sentence fragment with a particular word) was $14.88 \pm 7.6 \%$ for the first sentence (low constraint) and $89.1 \pm 9.2 \%$ for the second sentence (high constraint). These cloze probability patterns were assessed by presenting each individual sentence in isolation to 150 participants (Mestres-Missé et al., 2010). Only half of the pairs of sentences disambiguated multiple possible meanings, thus enabling the learning of the new word ( $\mathrm{M}+$ condition). For example, a duplet of sentences could be as follows: (1) "Every Sunday the grandmother went to the jedin"; (2) "The man was buried in the jedin." Using the context provided by the sentences, participants can infer and learn that jedin means "graveyard" as it is congruent with both the first and second sentence (Fig. 1A). Thus, participants were able to learn the meaning of up to 40 new words. For the other 40 pairs, the second sentences were scrambled so that they no longer matched their original first sentence. In this case, the new word was not associated with a congruent meaning across sentences and could not be correctly learned [e.g., (1) "Every night the astronomer watched the heutil," in which "moon" is one possible meaning of heutil; and (2) "In the morning break coworkers drink heutil," in which "coffee" is now one of the possible meanings of heutil, which is not congruent with the first sentence]. These sentences were part of a control condition $(\mathrm{M}-)$ designed to increase the level of difficulty of the task and to control for novelty during fMRI scanning (Ripollés et al., 2014) and was not analyzed in this work (note the nonsignificant correlation between the number of correctly learned $\mathrm{M}+$ words and the number of correctly rejected $\mathrm{M}-$ words; $r=-0.07, p>$ 0.66 ). In addition to the $\mathrm{M}+$ and $\mathrm{M}-$ conditions, nonreadable (NR) sentences (created by converting each letter of a sentence into a symbol) were also included in the paradigm. As is the case for $\mathrm{M}-$, this condition was created for fMRI purposes and is not analyzed. Before entering the scanner, participants were instructed to learn the meaning of a new word 

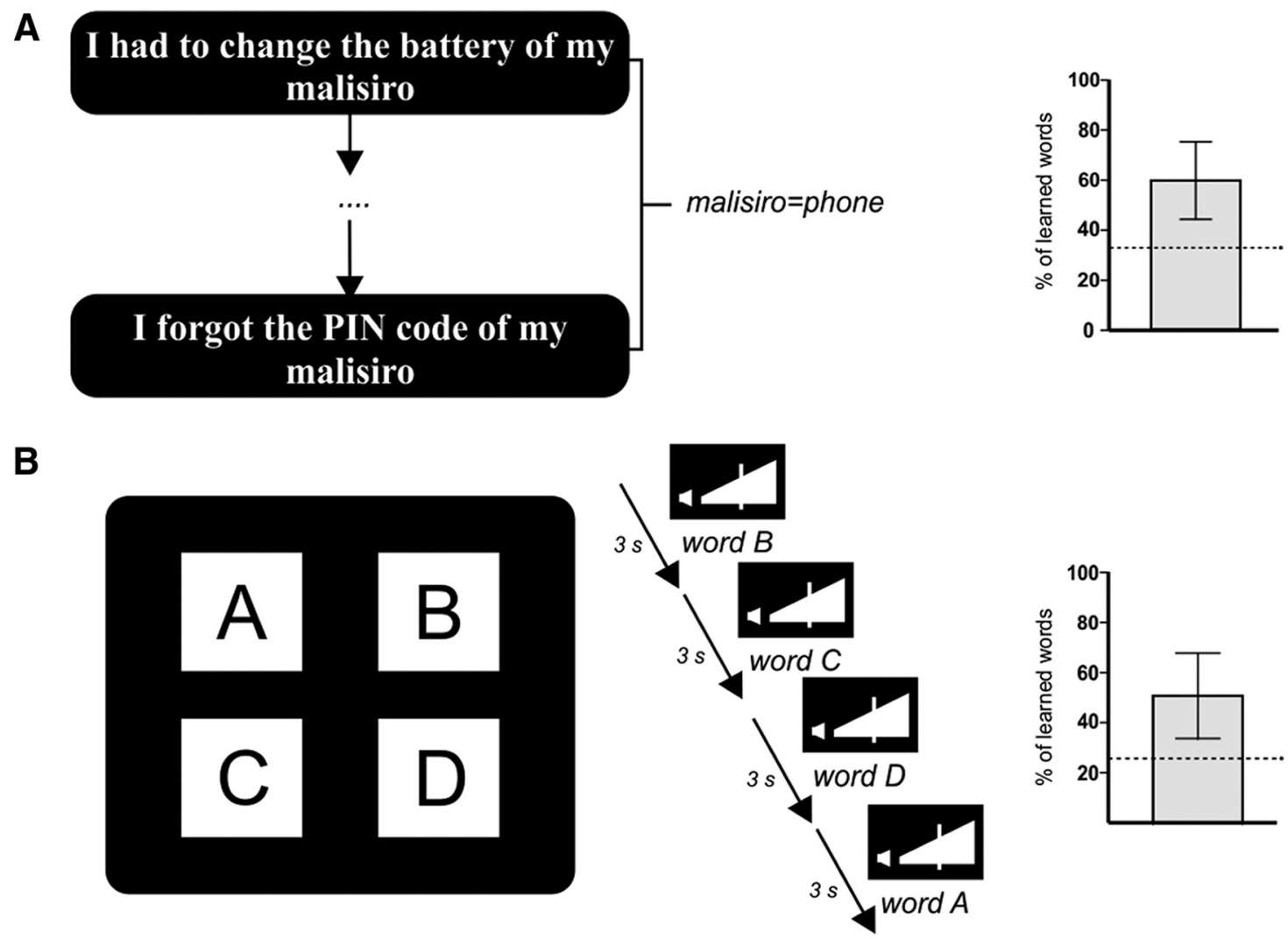

Figure 1. Schematic overview of the word-learning paradigms and behavioral results. The mean percentage of the number of learned words for each task is presented on the right $( \pm S D)$. Dotted lines indicate chance level. $\boldsymbol{A}$, Example of two congruent sentences for the $\mathrm{M}+$ condition of the CTXL paradigm. $\boldsymbol{B}$, Schematic example of a trial of the XSL task. Four unknown objects (A-D) are simultaneously shown on the screen while their corresponding words are aurally presented, in randomized order, every $3 \mathrm{~s}$.

only if both sentences had a redundant meaning $(\mathrm{M}+)$ and to reject the new words when learning was not possible $(\mathrm{M}-)$. To ensure that both stimulus types were equally comparable, participants were told that it was just as crucial to learn the words of the $\mathrm{M}+$ condition as it was to correctly reject the new words from the $\mathrm{M}-$ condition. For the NR conditions, participants were asked to look at the symbols and try to "read" them.

Four pairs of the $\mathrm{M}+$, four pairs of the $\mathrm{M}-$, and two pairs of the NR conditions were presented during a total of 10 short learning runs. Therefore, a total of 40 new words from the $\mathrm{M}+$ condition and 40 from the $\mathrm{M}$ - condition were presented during the whole experiment. To achieve an ecologically valid paradigm, the first and second sentences ending in the same new word were presented separately in time. The four first sentences of each of the $\mathrm{M}+$ and $\mathrm{M}-$ conditions (a total of eight new words) plus two "sentences" of the NR condition were presented in a pseudorandomized order (e.g., $\mathrm{M}+1 \mathrm{~A}, \mathrm{M}-1 \mathrm{~A}, \mathrm{M}-1 \mathrm{~B}, \mathrm{NR} 1 \mathrm{~A}, \mathrm{M}-1 \mathrm{C}$, $M+1 B, M+1 C, N R 1 B, M+1 D$, and $M-1 D)$ in each learning run. Later, the second sentence "pair" of both the $\mathrm{M}+$ and $\mathrm{M}-$ conditions was presented (i.e., the second presentation of the identical eight new words) in a pseudorandomized order including two sentences of the NR condition (e.g., $\mathrm{M}-2 \mathrm{C}, \mathrm{M}-2 \mathrm{~B}, \mathrm{NR} 2 \mathrm{~A}, \mathrm{M}+2 \mathrm{~B}, \mathrm{M}+2 \mathrm{D}, \mathrm{M}-2 \mathrm{D}, \mathrm{M}+2 \mathrm{C}$, $\mathrm{M}+2 \mathrm{~A}, \mathrm{M}-2 \mathrm{~A}$, and NR2B). The temporal order of the different new words in the first sentence presentation was not related in any systematic way to the order of presentation of the same new words in their second sentence. Each of the 20 trials of a run (10 first sentences and 10 second sentences) started with a $500 \mathrm{~ms}$ fixation cross and continued with the six first German words of the sentence presented for $2 \mathrm{~s}$ followed by a dark screen for $1 \mathrm{~s}$. Each new word was presented for $500 \mathrm{~ms}$ in the center of a black screen. All words were written in white using a font size of 22. Each new trial was preceded by a dark screen intertrial interval with a variable duration of 1-6 s (Poisson distribution; Hinrichs et al., 2000).

After each learning run, participants had to complete a brief recognition test. Participants were presented with a new word in the center of the screen and two possible meanings below, each on one side of the screen. In each test, all four $\mathrm{M}+$ and four $\mathrm{M}-$ new words presented during a learning run were tested in a pseudorandomized order. If the new word tested did not have a congruent meaning across the first and second sentences, and thus learning was not possible ( $\mathrm{M}-$ condition), participants had to press a button located in their left hand. In this case, the two possible meanings presented served as fillers: one was the meaning evoked by the second sentence of the $\mathrm{M}$ - new word being tested; the other word shown was the meaning evoked by another second sentence presented in the same run as the new word being tested. Instead, if the new word tested had a consistent meaning across the first and second sentences, and thus learning was possible $(\mathrm{M}+$ condition), participants had to select the correct meaning using a two-button pad placed on their right hand. In this case, one of the two possible meanings was correct and the other, which served as a filler, was the meaning of another new word presented in the same run. Therefore, the level of chance was set at 33\% accuracy as, for both the $\mathrm{M}+$ and $\mathrm{M}-$ conditions, three response options were available (no consistent meaning, consistent meaning on the left, consistent meaning on the right). As previously stated, the present work focused on the ability of the participants to learn new words and, therefore, only answers for the $\mathrm{M}+$ condition were analyzed (the $\mathrm{M}-$ condition was included for fMRI purposes). All participants completed a training block before entering the scanner to become familiarized with the task and the recognition test.

Cross-situational learning paradigm. For XSL, we used the exact same paradigm and parameters reported in previous research (Yu and Smith, 2007). The participants were required to learn the correct associations between 18 spoken pseudowords and their corresponding pictures, which were unknown objects. Insofar as the concept of meaning includes basic visual object representations (Gupta and Tisdale, 2009), this paradigm evaluates the ability to learn the correct word-to-meaning mappings through a cross-trial strategy, where within-trial referential ambiguity can be solved across multiple learning instances ( $\mathrm{Yu}$ and 
Smith, 2007). The learning set included 18 new words and 18 pictures. The spoken words were designed according to the phonotactical rules of the German language and were generated with the MBROLA speech synthesizer software (Dutoit et al., 1996), concatenating diphones at $16 \mathrm{kHz}$ from its German data base. In each learning trial, four object pictures appeared simultaneously on the screen while four bisyllabic words were presented, in randomized order, during $3 \mathrm{~s}$, for a total trial duration of $12 \mathrm{~s}$ (Fig. 1B). This configuration yielded a high within-trial referential ambiguity with four possible word-referent associations per learning trial. The participants were told that four words and four objects would co-occur on each trial and they were to learn which word goes with which object across trials. No information about word-picture correspondence was provided and the order of presentation of the words was not systematically related to the position of the objects. The task included 27 learning trials with a duration of $5 \mathrm{~min}$ and $24 \mathrm{~s}$. To generate each trial, four object-word pairs were randomly selected among the 18 pairs of the learning set. Each object appeared with its corresponding word a total of six times during the entire learning phase (i.e., six repetitions per objectword pair).

After the learning phase, the participants underwent a four-alternative forced-choice test. Each test trial presented one spoken word with four object pictures. Participants were requested to select the object that corresponded to the aurally presented word. Therefore, the level of chance was set at $25 \%$ accuracy. There were 18 test trials, 1 for each object-word pair presented during the learning phase. On each trial, the three filler objects were randomly selected from the set of 18 objects presented during the learning task.

Scanning parameters and diffusion measures. DW-MRI data were acquired on a $3 \mathrm{~T}$ scanner (MAGNETOM Verio using Syngo MR B17 software, Siemens) with a 32-channel phased-array head coil well suited for diffusion tensor imaging (DTI). Diffusion images were acquired with a twice-refocused, single-shot, spin-echo EPI sequence fully optimized (Reese et al., 2003) for DW-MRI of WM [72 axial slices; TR, 10400 ms; TE, 86 ms; GRAPPA (generalized autocalibrating partially parallel acquisitions) acceleration factor, 3 ; slice thickness, $2.0 \mathrm{~mm}$; acquisition matrix, $128 \times 128$; voxel size, $2.0 \times 2.0 \times 2.0 \mathrm{~mm}^{3}$ ]. Two runs with one nondiffusion-weighted volume (using a spin-echo EPI sequence coverage of the whole head) and 30 diffusion-weighted volumes (noncollinear diffusion gradient directions from Siemens MDDW mode; b-values, $1000 \mathrm{~s} / \mathrm{mm}^{2}$ ) were acquired. A high-resolution T1 (MPRAGE) image was also acquired during this MRI session (TR, $2500 \mathrm{~ms}$; TE, $4.82 \mathrm{~ms}$; TI, $1100 \mathrm{~ms}$; slice thickness, $1.0 \mathrm{~mm}$; acquisition matrix, $256 \times 256$; voxel size, $1.0 \times 1.0 \times$ $\left.1.0 \mathrm{~mm}^{3}\right)$.

Several diffusion measures can be extracted from DW-MRI. One of them is the radial diffusivity (RD) index, which has gained increasing interest in recent years. Several factors can contribute to the RD signal, including the number of axons and axon packing and diameter. Among all diffusion measures, $\mathrm{RD}$ has been consistently related to the myelin content along axons, with demyelination being associated with increased RD values (Song et al., 2002, 2005; Klawiter et al., 2011; Zatorre et al., 2012). Accordingly, thicker myelin sheaths have been related to increased conduction of action potentials along WM pathways (Fields, 2008). Indeed, in animal studies, directional measures (i.e., RD) — unlike summary parameters such as mean diffusivity or fractional anisotropy (FA) - provide better structural details of the state of the axons and myelin (Aung et al., 2013). Therefore, RD has been suggested to be a sensitive index of cognitive processing as fibers with greater myelination are hypothesized to enable a faster and more synchronized transfer of information between separated brain regions. This is of crucial importance to our hypothesis, as semantic learning requires the synergic cooperation of different cortical regions and $\mathrm{RD}$ values could provide an indirect measure of the speed of information exchange between language-related areas. Concordantly, a recent study (López-Barroso et al., 2013) showed a relationship between decreased RD values of the left long segment of the AF and the ability to learn new word forms from a continuous speech stream (i.e., new phonological word-form learning). Hence, for both manual and automatic dissections, RD maps for each participant were calculated using the eigenvalues extracted from the diffusion tensors, to be used later to calculate the microstructure of several WM tracts.
Manual dissection of WM pathways. Diffusion data processing started by correcting for eddy current distortions and head motion using the FMRIB Diffusion Toolbox (FDT), which is part of the FMRIB Software Library (FSL 5.0.1; www.fmrib.ox.ac.uk/fsl/; Jenkinson et al., 2012). Subsequently, the gradient matrix was rotated corresponding to the head movement to provide a more accurate estimate of diffusion tensor orientations using the fdt_rotate_bvecs program included in FSL (Leemans and Jones, 2009). In a next step, brain extraction was performed using the Brain Extraction Tool (Smith, 2002), which is also part of the FSL distribution. The analysis continued with the reconstruction of the diffusion tensors using the linear least-squares algorithm included in Diffusion Toolkit 0.6.2.2 [TrackVis software, Ruopeng Wang, Van J. Wedeen (trackvis.org/dtk), Martinos Center for Biomedical Imaging, Massachusetts General Hospital, Boston, MA]. As stated above, RD values were obtained.

Previously preprocessed DTI data were analyzed for deterministic tracking using a two-ROI approach within the TrackVis software. ROIs were defined using the FA and FA color-coded maps as a reference for individual anatomical landmarks (comparable to a T2-weighted MRI image). Here, we focused in WM pathways related to the ventral stream of language processing (Hickok and Poeppel, 2007; Rauschecker and Scott, 2009). Thus, we performed virtual in vivo dissections of the IFOF, ILF, and UF. We placed three spherical ROIs at the level of the anterior temporal lobe (temporal ROI), the posterior region located between the occipital and temporal lobe (occipital ROI), and the anterior floor of the external/extreme capsule (frontal ROI). To define each of the tracts of interest, we applied a two-ROI approach. The ILF was obtained by connecting the temporal and occipital ROIs. The streamlines passing through the occipital lobe and frontal ROIs were considered as part of the IFOF. The frontal capsule ROI was united to the temporal ROI to delineate the UF. All these ROIs were applied according to a well defined anatomical atlas (Catani and Thiebaut de Schotten, 2008). The exclusion of single fiber structures that do not represent part of the dissected tract was achieved using subject-specific non-ROIs. These processes were performed for both the left and the right hemisphere. After the dissection was completed, the mean $\mathrm{RD}$ value of each tract was extracted for further analysis.

Although this work is focused in the ventral stream of language processing, the long segment of the AF (the main tract associated to the dorsal stream of language processing) was also dissected as a control. This approach was based on previous research relating the long segment of the AF to phonological word learning, when novel word forms without meaning are segmented from continuous speech (López-Barroso et al., 2013). This segment was dissected using established guidelines and a two-ROI approach (Catani et al., 2005; López-Barroso et al., 2013; François et al., 2016; Vaquero et al., 2017). A first frontal ROI was placed in the coronal plane between the central fissure and the cortical projection of the tract. A second temporal ROI was placed in the axial plane involving the fibers descending to the posterior temporal lobe through the posterior portion of the temporal stem. The streamlines going through the frontal and temporal ROIs were classified as the long segment of the AF. This process was performed for both the left and the right hemisphere.

Automatic dissection of WM pathways. For the automatic virtual dissection, we used the Automatic Fiber Quantification (AFQ; https:// github.com/jyeatman/AFQ) software, which can identify 18 major WM tracts and allows for the calculation of differentiated diffusion measurements along the whole dissected pathways (Yeatman et al., 2012). AFQ was run under MATLAB version R2012a (MathWorks). Data were first preprocessed using the mrDiffusion toolbox (http://web.stanford.edu/ group/vista/cgi-bin/wiki/index.php/MrDiffusion). This preprocessing included standard steps (motion correction of the DW-MRI images, coregistration of the DW-MRI images to the T1, realignment to the anterior commissure-posterior commissure line, and tensor calculation). The preprocessed DW-MRI data were then fed to the AFQ standard pipeline consisting of the following three main steps: (1) whole-brain tractography; (2) tract segmentation; and (3) fiber tract refinement (Yeatman et al., 2012). Whole-brain tracking used a deterministic streamlines tracking algorithm with a fourth Runge-Kutta path integration method and $1 \mathrm{~mm}$ fixed step size (Mori et al., 1999; Basser et al., 2000). Tract segmen- 
tation was performed using a two-ROI approach, using ROIs defined in MNI space (Wakana et al., 2007). These MNI ROIs were registered to each participant's space by means of nonlinear transformation (Friston and Ashburner, 2004). Only fibers passing through the two specified ROIs were assigned to a particular tract. Fiber tract refinement was accomplished in two phases. For each participant, the dissected tracts in native space were first compared with a probability atlas of major WM pathways (also registered from standard to native space; Hua et al., 2008), and aberrant fibers were discarded. Second, the fibers that spatially deviated $>3$ or 4 SDs from the core or the tract were also removed (we adjusted the value depending on the subject and tract until aberrant fibers were left out). Finally, diffusion properties were extracted for the AF, IFOF, ILF, and UF. Specifically, RD values at 100 equidistant nodes along each tract were calculated. This approach enabled the assessment of the relationship between each participant's word-learning ability and the WM microstructure on a point-by-point basis along each of the dissected tracts (i.e., using the 100 different $\mathrm{RD}$ values obtained per WM tract).

In addition, for the automatic method and for visualization purposes only, we created approximated probabilistic group overlaps of the AF, ILF, UF, and IFOF. First, the coordinates for each of the 100 nodes that form each AFQ tract were extracted for each participant. Then, $3 \mathrm{~mm}$ spheres were created for each of the nodes using MarsBar (Brett et al., 2002). For each tract and subject, the spheres were combined together and binarized to obtain an approximated map of the AFQ-dissected WM pathway in native space. The structural high-resolution T1-weighted images of each subject were then coregistered to the individual diffusion maps by using Statistical Parametric Mapping software (SPM8; Wellcome Department of Imaging Neuroscience, University College, London, UK; www.fil.ion.ucl.ac.uk/spm). New Segment (Ashburner and Friston, 2005) was applied to the T1 images to obtain gray and white matter tissue probability maps that were imported and fed into Diffeomorphic Anatomical Registration using Exponentiated Lie algebra (DARTEL; Ashburner, 2007). The flow fields obtained from this process were applied to the tract masks in native space previously created with MarsBar to register them to MNI space. Finally, for each tract, all of the MNI individual tract masks were averaged to obtain an approximated probabilistic map of the dissected WM pathways. For display purposes, we thresholded these maps at a 50\% threshold (showing only voxels that are part of the tract in at least half of the participants). Moreover, to compare these WM pathways with the cortical regions usually associated to semantic processing, we conducted a meta-analysis using NeuroSynth (a platform for large-scale, automated meta-analysis of fMRI data; www. neurosynth.org; Yarkoni et al., 2011). We calculated a term-based search on "semantic" that resulted in 884 studies (search performed on 23 April 2017). Then, a reverse inference map was generated in MNI space. This reverse inference map depicts the brain regions that are preferentially related to the term semantic (i.e., it shows areas that are more diagnostic of the term semantic, instead of brain regions that are just activated in studies associated with that term). Finally, the probabilistic tract masks were visually compared with the cortical fMRI semantic-related activations (François et al., 2016).

Experimental design and statistical analysis. Correlational analyses were performed using MATLAB version R2012a. For all correlations computed regarding manual dissections, $\mathrm{RD}$ mean values of manual tracts $>2.5$ SDs of the mean were considered outliers and removed (LópezBarroso et al., 2013). AFQ was occasionally unable to dissect a particular tract; therefore, the affected WM pathway was excluded from all analyses regarding automatic dissections (one right AF, one left and right ILF, one left and right UF, one left IFOF, and four right IFOFs). To compare manual and AFQ dissections, Spearman's rank-order correlations were first computed for mean RD values of each tract of interest (for automatic dissections, mean $\mathrm{RD}$ values were calculated as the average of the $100 \mathrm{RD}$ values obtained).

To assess the relationship between word learning and the WM microstructure, Spearman's rank-order correlations were also used to correlate scores in both word-learning tasks and RD values. For manual dissections, scores from both the CTXL and the XSL tasks were correlated with the mean RD values of the ILF, UF, IFOF, and the long segment of the AF from both hemispheres. False discovery rate (FDR) correction was used to control for multiple comparisons ( 16 correlations: 4 tracts $\times 2$ hemispheres $\times 2$ word-learning scores; for a similar approach, see KronfeldDuenias et al., 2016; Rojkova et al., 2016; Zhao et al., 2016; Vaquero et al., 2017).

Regarding automatic dissections, correlations were computed between the scores of both learning tasks and the $100 \mathrm{RD}$ values obtained for the ILF, UF, IFOF, and the AF from both hemispheres. To correct for multiple comparisons (100 correlations were computed per tract and score), we used an FWE-corrected cluster size threshold, calculated by a nonparametric permutation method (Nichols and Holmes, 2002). Specifically, we used the AFQ_MultiCompCorrection function (which is part of the AFQ software; Yeatman et al., 2012), which returns, among other values, the minimum number of significant and sequential nodes (i.e., a cluster) so that any cluster with a greater number of significant nodes is considered corrected for multiple comparisons. We, thus, only report those clusters in which a significant correlation at an uncorrected level of $p<0.05$ occurred in a sufficient number of sequential nodes (for similar approaches, see Dodson et al., 2017; Travis et al., 2016; Vaquero et al., 2017).

Finally, we used a cross-validation approach to assess whether the white matter tracts showing significant correlations between diffusion values and word-learning scores actually predicted (instead of just correlated with) CTXL and XSL (for a similar analysis, see King et al., 2016). Note that for this analysis we only used data from the automatic method, as it allowed us to use all the information provided by the AFQ software (the 100 diffusivity values along each tract) to fit a generalized linear model (GLM), as opposed to the correlational analysis in which we used each single value separately to calculate 100 correlations with the learning scores. In this analysis, we expected CTXL and XSL ( $y$ in a GLM) to be a linear combination of the 100 diffusivity values obtained per tract $(x 1 \ldots x 100$ in a GLM). In other words, we tried to predict a vector of CTXL or XSL scores $(y)$ from a matrix of diffusivity values (X: 100 diffusivity values $X N$ participants). All analyses were performed using the MNE (version 0.15; Gramfort et al., 2013, 2014) and Scikit-Learn packages (Pedregosa et al., 2011).

To do this, for each tract, we first fitted a linear model to a training subset of $75 \%$ of the available data (e.g., AFQ was able to automatically dissect the left AF of all 40 participants; in this case, the training subset came from the 100 diffusivity values of 30 participants). To fit the model, we used a ridge regression to better account for multicollinearity effects (as there is a linear relationship between the 100 diffusivity values) and also to prevent overfitting, since we have more features (100 diffusivity values) than observations (40 participants). Using the parameters obtained by this fit, we then predicted word-learning scores on a different testing subset formed by the remaining $25 \%$ of the data (e.g., the 100 diffusivity values of the remaining 10 participants in the left AF example above). These predicted scores could then be compared with the real CTXL and XLS scores (i.e., the ground truth). We did so by computing a Spearman correlation between the real and the predicted values (King et al., 2016), which resulted in an $r_{s}$ value: the closer this value is to 1 , the more similar the predicted and the real word-learning scores are. This analysis was repeated 100 times per tract using a random permutation cross-validator (ShuffleSplit function from the Scikit-Learn package; we created random subdivisions of our population into training and testing subsets). In other words, we generated 100 training (with $75 \%$ of available participants) and test (with $25 \%$ of available participants) random subsets that were used for the following: (1) to fit a model (using the training subset); (2) to predict the word-learning scores (using the testing subset); and (3) finally, to generate an $r_{s}$ value (using the predicted and the real scores of the training set). We then averaged the $100 r_{s}$ values to obtain a mean measure of the goodness of fit of the prediction made by the diffusion properties (the 100 diffusivity values) of each tract.

To estimate the null distribution of each prediction, we repeated the same approach 10,000 times after randomly shuffling the $y$ label (e.g., CTXL or XSL scores) while maintaining the $X$ (100 diffusivity values $\times N$ participants) constant. Thus, in each of the 10,000 permutations, each $X$ set of values (the 100 diffusivity nodes) is randomly paired with a $y$ value (i.e., a word-learning score), and we expect the correlation between the predicted and the real values of the testing set to be 0 (the model is fit 
Table 1. Spearman $r_{s}$ and $p$ values for each correlation between mean RD values of pair of tracts in each hemisphere

\begin{tabular}{lllr}
\hline & Left hemisphere & Right hemisphere & Difference \\
\hline AF-IFOF & $r_{s}=0.53, p<0.001$ & $r_{s}=0.39, p<0.017$ & 0.14 \\
AF-ILF & $r_{s}=0.38, p<0.012$ & $r_{s}=0.35, p<0.037$ & 0.03 \\
AF-UF & $r_{s}=0.37, p<0.021$ & $r_{s}=0.44, p<0.007$ & -0.07 \\
IFOF-ILF & $r_{s}=0.68, p<0.001$ & $r_{s}=0.58, p<0.001$ & 0.10 \\
IFOF-UF & $r_{s}=0.48, p<0.002$ & $r_{s}=0.47, p<0.003$ & 0.01 \\
UF-ILF & $r_{s}=0.51, p<0.001$ & $r_{s}=0.37, p<0.021$ & 0.13 \\
\hline
\end{tabular}

All correlations survived a $p<0.05$ FDR-corrected threshold.

using an incorrect labeling). We finally ranked the 10,000 random $r_{s}$ values obtained (i.e., created from random $X-y$ pairings), so that any real $r_{s}$ (coming from the original data; created using correct $X-y$ pairings) with a value greater than that of the 9500 position in the ranking allowed us to reject the null hypothesis with a $p$ value $<0.05$ (i.e., reject that there is no correlation between the predicted and the real learning scores). Thus, this method allowed us to assess whether the relationship between the predicted and the real word-learning scores was significant: whether a particular tract is a good predictor of a particular word-learning score.

\section{Results}

\section{Behavioral results for the semantic learning tasks}

Participants showed above-chance performance in both tasks as they learned $60 \pm 15.51 \%$ of the new words in the CTXL para$\operatorname{digm}\left(\mathrm{M}+\right.$ condition; $_{(39)}=10.80, p<0.001$; chance level was at $33 \%$, see Materials and Methods) and $52 \pm 17 \%$ of the objectword pairs in the XSL task $\left(t_{(39)}=9.97, p<0.001\right.$; chance level was at 25\%; see Materials and Methods). This last result replicates the learning performance reported in a previous study using the same task ( $\sim 55 \%$ of accuracy; Yu and Smith, 2007). No behavioral outliers $(>2.5$ or $<2.5$ SDs) were detected in any of the tasks. A significant association was observed between the performance of both learning tasks $(r=0.45, p<0.005)$. Note that, despite this correlation between behavioral measures, their neural underpinnings differed significantly (see below).

\section{Manual and automatic virtual dissections}

For manual dissections and to characterize the hemispheric particularities of the dissected tracts, we first computed laterality indices (LIs; we used tract volume; Thiebaut de Schotten et al., 2011). LIs range from -1 to 1 , with more positive values representing left lateralized structures [calculated as (LeftVolume RightVolume)/(LeftVolume + RightVolume)]. Our results replicate previous findings (Thiebaut de Schotten et al., 2011a; López-Barroso et al., 2013) with the AF ( $\mathrm{LI}=0.19 \pm 0.34)$ being the only tract showing a left lateralized pattern (IFOF $=0.07 \pm$ $0.19 ; \mathrm{ILF}=-0.08 \pm 0.19 ; \mathrm{UF}=-0.07 \pm 0.17)$. In addition, we also calculated Spearman rank correlations between intrahemispheric tracts (correlations between RD values of tracts of the same hemisphere). Results (Table 1) show that, in general, $r_{s}$ values were larger for correlations between left-hemispheric tracts, especially for the left AF-IFOF and left ILF-UF pairs.

Regarding the direct comparison between manual and automatic dissections, there was a significant correspondence between the mean $\mathrm{RD}$ values of both approaches for all the tracts of interest, with almost all $r_{s}$ values $>0.60$, indicating a strong agreement between methods (left AF, $r_{s}=0.69$; right AF, $r_{s}=0.86$; left $\mathrm{UF}, r_{s}=0.52$; right UF, $r_{s}=0.63$; left ILF, $r_{s}=0.60$; right ILF, $r_{s}=0.53$; left IFOF, $r_{s}=0.63$; right IFOF, $r_{s}=0.66$; all $p$ values $<0.001)$. The coefficients obtained for the left AF are similar to those reported in previous research (Vaquero et al., 2017), which also compared manual dissections of the left long segment of the
AF with automatic fiber tracking. This shows that, considering the manual dissections as the gold standard, automatic dissections produced reliable diffusion measures of microstructural WM integrity, further corroborating the validity of the anatomical measures used here.

\section{Relationship between learning scores and structural connectivity of language-related pathways: manual dissections}

To address the critical question on the relationship between WM structure and word-learning success, we then correlated subjectspecific WM measures with individual learning success. We used $\mathrm{RD}$ values (for both the automatic and manual correlations) as a measure of WM microstructure, as RD has been consistently related to the myelin content along axons-an indicator of increased conduction of action potentials (Song et al., 2002, 2005; Fields, 2008; Klawiter et al., 2011; Zatorre et al., 2012)—and thus could serve as a sensitive index of cognitive processing. While the WM microstructure of several pathways crossing the temporal lobe (especially those of the left hemisphere) showed significant relationships with both learning performances at an uncorrected threshold, only the left ILF and the left UF were significantly associated with CTXL (18.5\% of the variance explained by the left ILF) and XSL (33.6\% of the variance explained by the left UF), when corrected for multiple comparisons (Fig. 2, see Table 2 for all correlation coefficients). Importantly, both the left and right long segments of the AF failed to show a significant relationship (corrected or uncorrected) with performance on any of the semantic learning paradigms. Hence, our results reveal that the pattern of behavior is tightly linked to the integrity of WM pathways in the temporal lobe of the left hemisphere.

Given that the IFOF has previously been implicated in semantic processing (Duffau et al., 2005, 2009; Mandonnet et al., 2007), we conducted further analyses to test for this hypothesis. In particular, we computed a hierarchical multiple regression in which the IFOF was added as an independent predictor to a model that included the ILF (with CTXL as a dependent variable) or the UF (with XSL as dependent variable). Adding the IFOF failed to significantly increase the amount of variability predicted by the model in both cases (XSL: $0.4 \%$ increase in $R^{2} ; F_{(1,34)}=0.197$; $p=0.66$; tolerance $=0.76$; variance inflation factor $=1.31$; CTXL: $0.1 \%$ increase in $R^{2} ; F_{(1,35)}=0.06 ; p=0.808$; tolerance $=$ 0.438 ; variance inflation factor $=2.28$ ).

\section{Relationship between learning scores and structural connectivity of language-related pathways: automatic dissections}

As stated before, the automatic tracking method allows for the extraction of individual $\mathrm{RD}$ values along each of the tracts of interest (right/left AF, IFOF, UL, and ILF). Thus, the correlations between word-learning scores and RD values were calculated on a point-by-point basis using 100 nodes equidistantly placed along each tract. Figure 3 shows an approximate group map of the four different tracts dissected with the automatic procedure in MNI space (a voxel is shown if it was part of a tract in at least $50 \%$ of the participants; see Materials and Methods), along a meta-analysis of semantic-related fMRI activity calculated using NeuroSynth (Yarkoni et al., 2011). This meta-analysis revealed, as expected, a strongly left-lateralized cortical network, covering not only the left IFG, anterior temporal lobe, and pMTG, but also the inferior temporal and the fusiform gyri, the angular gyrus, and even mesial temporal regions such as the hippocampus and parahippocampus. To further assess the reliability of the tracts dissected 

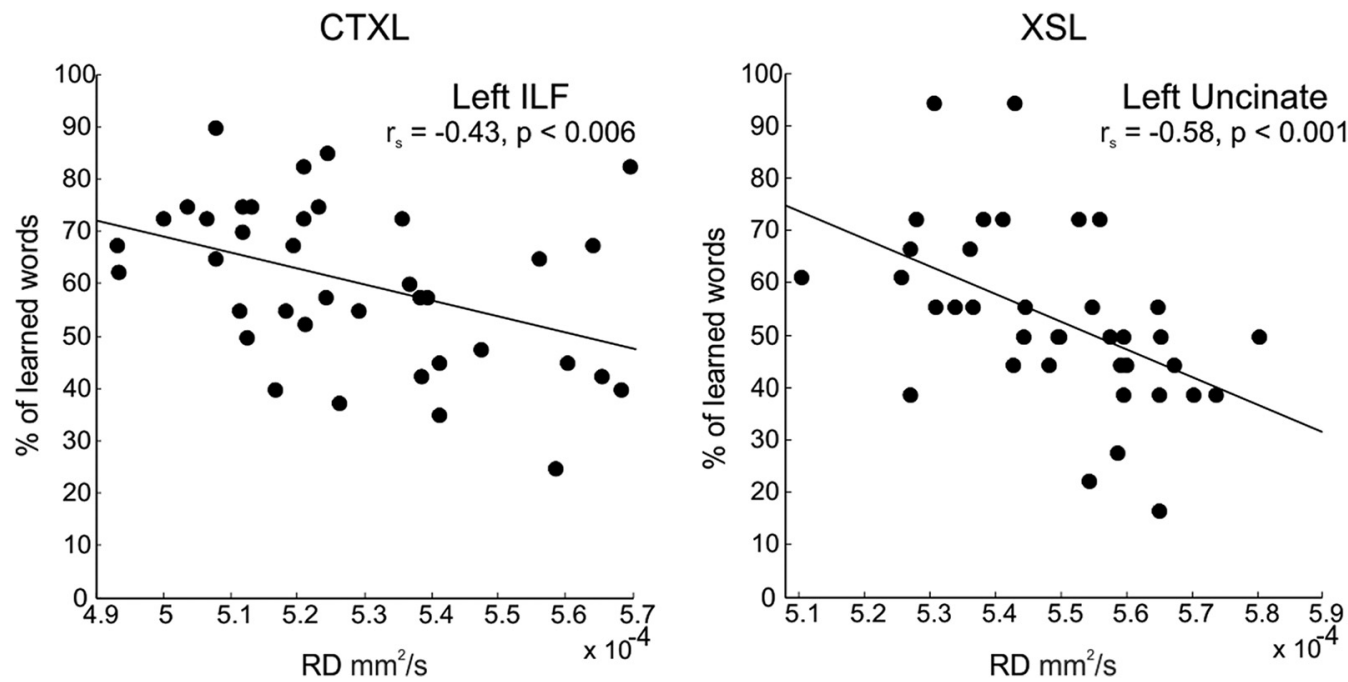

Figure 2. Manual dissections. Scatter plots of the two correlations showing a significant $p<0.05$ FDR-corrected result. WM microstructure of the left ILF correlated with the percentage of learned words during (TXL (left), while diffusion values of the left UF correlated with the percentage of learned words during XSL (right). Lower RD values indicate better WM microstructure.

Table 2. Spearman $r_{s}$ and $p$ values for each correlation between word-learning success in the two different learning paradigms and the mean RD value of each tract of interest

\begin{tabular}{|c|c|c|}
\hline$\overline{W M}$ & CTXL & XSL \\
\hline \multicolumn{3}{|l|}{ Dorsal } \\
\hline \multicolumn{3}{|l|}{$\mathrm{AF}$} \\
\hline Left & $r_{s}=-0.18, p>0.266$ & $r_{s}=-0.21, p>0.183$ \\
\hline Right & $r_{s}=-0.08, p>0.623$ & $r_{s}=-0.18, p>0.283$ \\
\hline \multicolumn{3}{|l|}{ Ventral } \\
\hline \multicolumn{3}{|l|}{ ILF } \\
\hline Left & $r_{s}=-0.43, p<0.006$ & $r_{s}=-0.32, p<0.049$ \\
\hline Right & $r_{s}=-0.33, p<0.038$ & $r_{s}=-0.13, p>0.403$ \\
\hline \multicolumn{3}{|l|}{ UF } \\
\hline Left & $r_{s}=-0.25, p>0.131$ & $r_{s}=-0.58, p<0.001$ \\
\hline Right & $r_{s}=-0.34, p<0.036$ & $r_{s}=-0.28, p>0.087$ \\
\hline \multicolumn{3}{|c|}{ 's } \\
\hline Left & $r_{s}=-0.35, p<0.027$ & $r_{s}=-0.35, p<0.027$ \\
\hline Right & $r_{s}=-0.17, p>0.300$ & $r_{s}=-0.20, p>0.216$ \\
\hline
\end{tabular}

Notice that lower RD values indicate better WM microstructure integrity. Correlations in bold survived a $p<0.05$ FDR-corrected threshold.

using $\mathrm{AFQ}$, we also compared our MNI group-specific probabilistic tracts (Fig. 3) with a well known probabilistic tractography atlas (Thiebaut de Schotten et al., 2011a,b, 2014). We thresholded both our probabilistic group-specific maps and those of the probabilistic atlas at the $50 \%$ level (i.e., showing only voxels that were part of a particular tract in at least half of our participants). We then calculated the percentage of voxels of our tracts that overlapped with the atlas. As expected, there was a great overlap between all the dissected tracts for our group and their corresponding counterparts in the atlas (left AF, $81.91 \%$; left IFOF, 80.70\%; left ILF, $85.89 \%$; left UF, $74.34 \%$; right AF, $85.59 \%$; right IFOF, 83.59\%; right ILF, 99.19\%; right AF, 89.67\%). These results add further evidence that the obtained dissections are anatomically plausible and reliable.

Regarding the correspondence between semantic learning measures and automatic dissections, two clusters along the left ILF (located in the posterior and in the anterior to mid-portion of the tract) showed significant FWE-corrected associations with individual word-learning success in the CTXL paradigm (Fig. 4A; $32.8 \%$ of the variance explained by the peak node at the left ILF). The cluster with the strongest WM-behavior correlation was the one located posteriorly, around the pMTG (Fig. 4C, approximate location of this cluster in MNI space). In addition, one cluster at the anterior to mid-region of the ILF also showed a significant association with XSL (Fig. 4B; $21 \%$ of the variance explained by the peak node at the left ILF). Importantly, there was an overlap between the clusters showing significant correlations for both XSL and CTXL at the anterior to mid-section of the ILF (near the hippocampus; Fig. 4C, light blue cluster). To quantify this overlap, we calculated a dice similarity index (DSI; Dice, 1945), which we defined as twice the overlap between the number nodes correlating with CTXL and XSL at the same time, divided by the sum of the number of nodes in the anterior to mid-portion of the ILF that correlate with CTXL and XSL independently (Seghier et al., 2008; Ripollés et al., 2012). DSI ranges between 0 and 1 (0, no overlap; 1 , perfect similarity) with values exceeding 0.80 considered as an indicator of a high similarity (Wilke et al., 2011). The DSI for this cluster was 0.89 , which indicates a high overlap between the nodes located in the anterior to mid-part of the ILF that correlates both with XSL and CTXL.

The only other tract showing significant correlations with the semantic tasks was the UF. A significant FWE-corrected cluster correlating with XSL scores was found in the inferior part of the left UF (Fig. $5 A, B ; 30.3 \%$ of the variance explained by the peak node at the left UF; the nonsignificant correlations with CTXL outcomes are also shown for the sake of completeness). This significant cluster is placed around the left anterior temporal lobe (Fig. 5C, approximate location of this cluster in MNI space).

Finally, we applied a cross-validation approach to assess whether the left ILF and left UF actually predicted (instead of just correlated with) CTXL and XSL scores (King et al., 2016). Results for the left AF and left IFOF are also presented as controls. Figure 6 depicts the mean and SD of $r_{s}$ values that describe the goodness of fit of each tract as a predictor for each word-learning score (the $r_{s}$ value is computed between the predicted and the real learning scores; the greater the $r_{s}$ value is, the more similar these values are and the better the prediction). As expected, both the left ILF and UF were significantly predictive of CTXL and XSL, respectively. In addition, the left UF was also a good predictor of CTXL, while the $r_{s}$ value between the predictions made by the left ILF RD values and the real XSL scores approached significance (probability of rejecting the null hypothesis that $r_{s}=0$ was 0.0789 ). This 


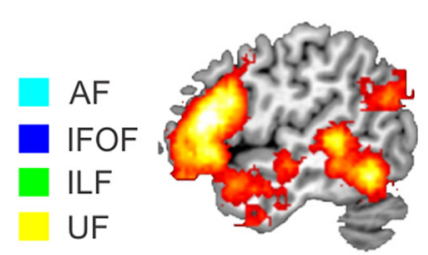

L $x=-50$

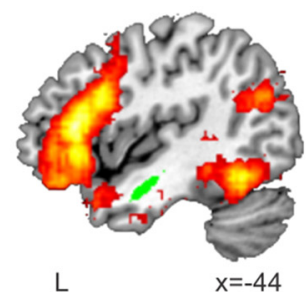

$\mathrm{L}$
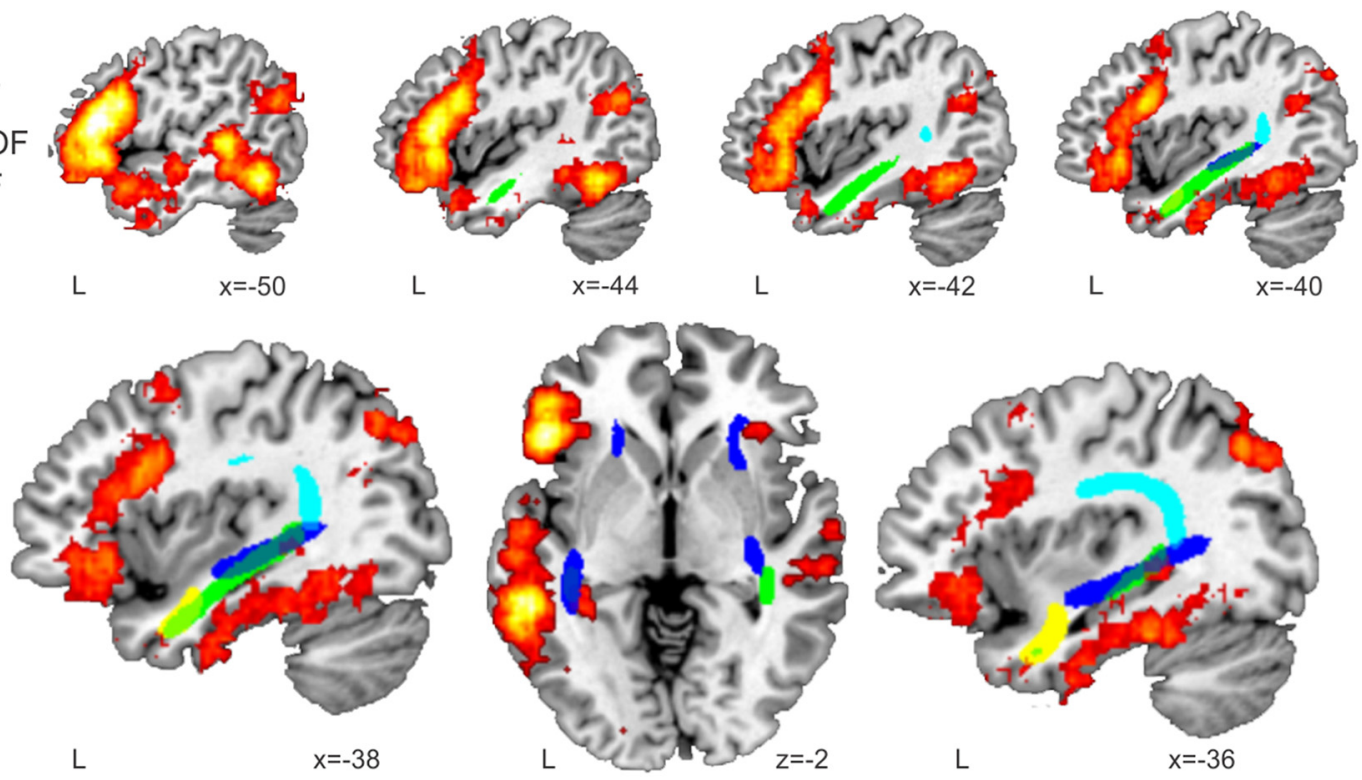

L

$x=-38$

L
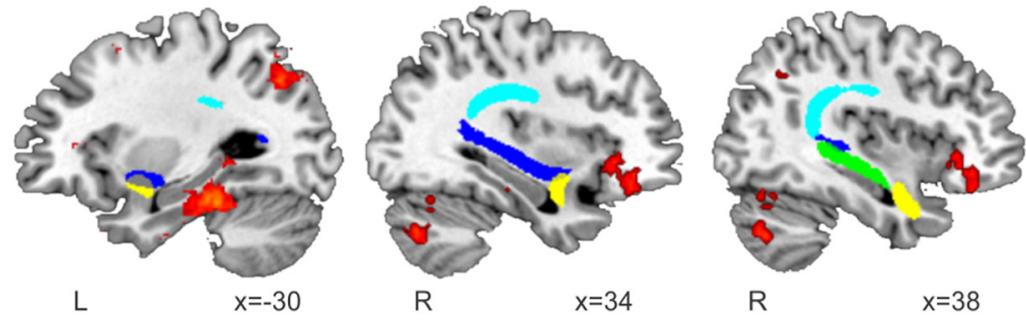

L

$x=-34$

L

$\mathrm{R}$

$x=34$

R

$x=38$

Figure 3. Mean tracts produced by automatic dissections and the semantic network. An approximation of the mean tracts dissected automatically (thresholded at $50 \%$ probability; see Materials and Methods) is shown (light blue, AF; dark blue, IFOF; green, ILF; yellow, UF). In red-yellow, thresholded at a $p<0.01$ FDR-corrected threshold, the NeuroSynth fMRI meta-analysis on the term semantic is shown. Results are overlaid over a canonical T1 with MNI coordinates at the bottom right of each slice. Neurological convention is used. L, Left hemisphere; $R$, right hemisphere.

further shows that not only was there a significant relationship between the left ILF and UF tracts and CTXL and XSL scores, but also that the pattern of RD values from these tracts actually predicted word learning.

\section{Discussion}

The goal of the present study was to assess whether the microstructural anatomy of the ILF, UF, and IFOF was associated with individual differences in semantic learning for different types of word-to-meaning mappings (CTXL and XSL). Our results indicate that individual differences in the microstructure of the left ILF and UF predict semantic learning success beyond their suggested-and still controversial (Duffau et al., 2005, 2009; Mandonnet et al., 2007)_contribution to semantic processing. Specifically, the manual dissection approach indicated that the left ILF and the left UF correlated with scores in CTXL and XSL, respectively (Fig. 2). The automatic dissections further corroborated these findings, but revealed - despite both tasks being correlated on the behavioral level_-only a partly overlapping WM network: although the anterior to mid-section of the ILF correlated with both learning tasks, its posterior region (near the pMTG) was more associated with CTXL (Fig. 4C). In the same line, the anterior inferior portion of the left UF (around the anterior temporal lobe) significantly correlated with XSL (Fig. 5C). Remarkably, the cross-validation analyses showed that both the left ILF and UF were good predictors of performance on both semantic learning tasks, although, as expected, the left ILF generated better predictions than the left UF for CTXL and the opposite occurred for XSL (Fig. 6). No significant correlations were found for the long segment of the AF, part of the dorsal pathway for phonological processing (Hickok and Poeppel, 2007; Rauschecker and Scott, 2009). Previous research, using the same CTXL task (Ripollés et al., 2014) and similar learning paradigms (Rodríguez-Fornells et al., 2009), shows that contextual learning is supported by regions related to semantic processing (Lau et al., 2008; Binder et al., 2009; Fig. 3), which are connected by the WM fiber tracts of the ventral pathway of language processing (ILF, UF, and IFOF): the left ventral IFG (BA 47), the pMTG, and the ATL. The left ventral IFG has been related to semantic processing, with a special role in guiding top-down retrieval of semantic knowledge from long-term memory (Fiez, 1997; Badre et al., 2005; Badre and Wagner, 2007). The pMTG has been suggested to be an important hub for semantic processing (Turken and Dronkers, 2011), which could also act as a lexical interface that mediates mapping between phonetic representations in superior temporal regions and semantic features widely localized over a distributed network (Hickok and Poeppel, 2007; Rodriguez-Fornells et al., 2009; Gow, 2012). In line with this view, our results revealed the highest correlations with CTXL for the WM surrounding the pMTG (Fig. 4C). Together, these findings suggest that the combined activity of the above-mentioned regions and the temporal WM pathways connecting them-especially by the ILF around the pMTG-allowed learners (1) to retrieve the possible meaning candidates that cohere with the contextual information available in a learning instance and (2) to narrow down the alternative meanings to finally learn the correct word-to-meaning mappings. 

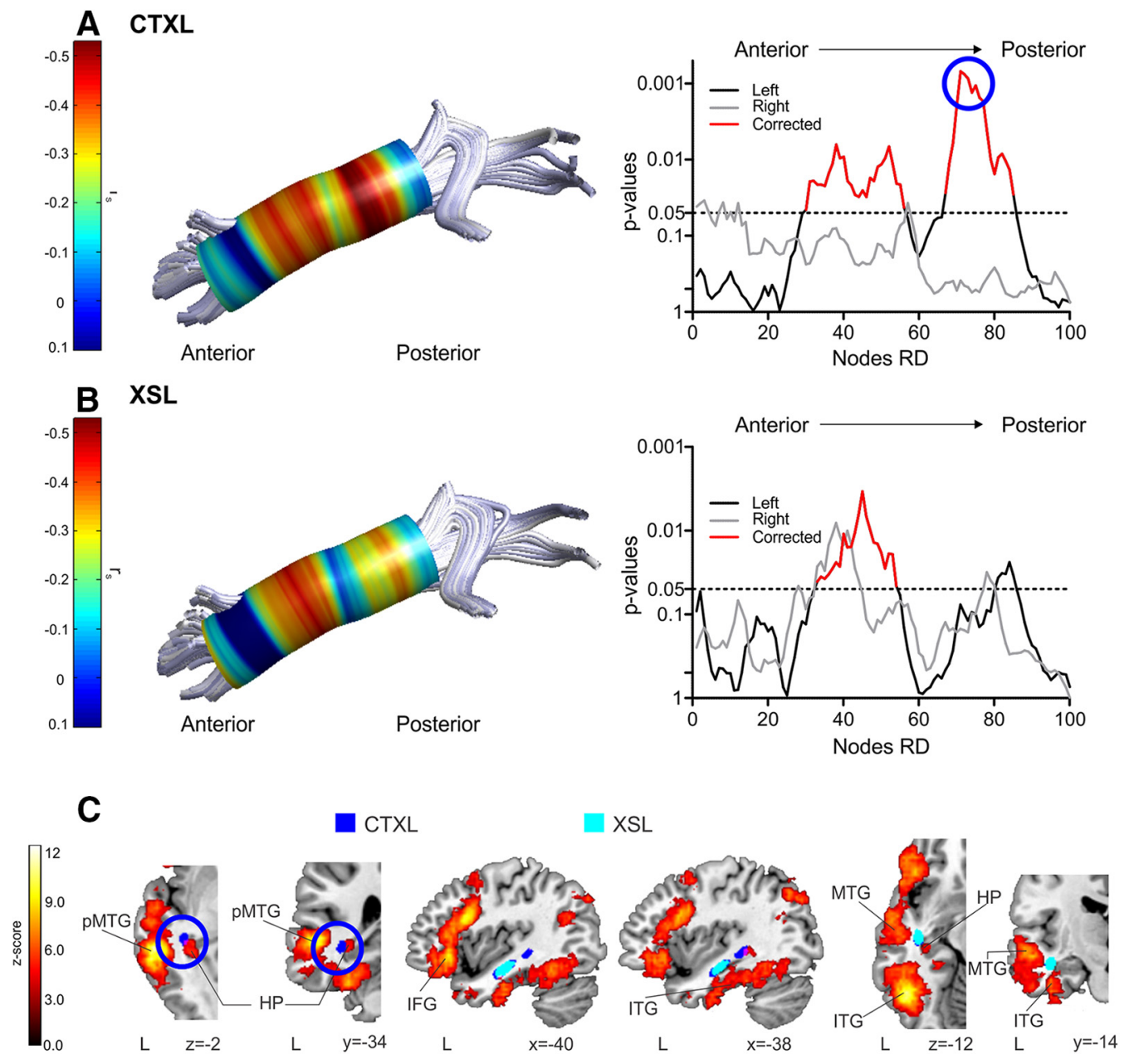

Figure 4. ILF automatic dissections. Correlations between word-learning scores and RD values were calculated on a point-by-point basis using 100 nodes equidistantly placed along the ILF. In $\boldsymbol{A}$ and $\boldsymbol{B}$, a tract from an individual subject with a colored overlay representing the Spearman's rank-order $r_{s}$ value is shown on the left. The $p$ values associated with each $r_{s}$ value at each of the 100 points extracted with the automatic procedure are displayed on the right (left hemisphere in black; right hemisphere in gray; FWE-corrected clusters in red). $\boldsymbol{A}$, Results of the correlations between the WM microstructural properties of the left ILF and individual success in CTXL. B, Results of the correlations between the WM microstructural properties of the left ILF and individual success in XSL. C, Approximate location in MNI space of the results shown in $\boldsymbol{A}$ and $\boldsymbol{B}$ (see Materials and Methods), shown over a canonical T1 along a Neuro-Synth meta-analysis of semantic-related fMRI activity. Neurological convention is used, with MNI coordinates shown at the right bottom of each slice. In dark blue, portions of the ILF correlating with CTXL are shown. In light blue, portions of the ILF correlating with XSL are shown. Note the overlap at the anterior to mid-section of the ILF. The cluster at the posterior region of the ILF is the one showing the greatest correlation (see blue circle in A) for CTXL (highlighted also with a blue circle in axial and coronal slices). L, Left hemisphere; HP, hippocampus; ITG, inferior temporal gyrus.

Learning the correct word-to-meaning mappings in a situation of referential ambiguity can be also achieved through the computation of cross-situational statistics (Smith et al., 2014). It has been proposed that XSL is supported by statistical learning mechanisms where word learning takes place gradually by tracking the frequency of co-occurrence between many words and referents in a cross-trial approach (Yu and Smith, 2007). Thus, during XSL, we expected participants to use different strategies than those used during CTXL. Our results for the automatic dissections show that the neuroanatomy of both the left ILF and UF was predictive of XSL scores. This time, the area showing the highest correlations (Fig. 5) was the WM surrounding the left ATL. Note that there is only a partial overlap for WM correlations for both CTXL and XSL in the anterior to mid-section of the ILF. Although fMRI studies focusing on XSL are lacking, the left ILF has been related to visual object recognition (Mummery et al., 1999), possibly playing a role in linking object representations and labels. Importantly, the UF has been related to lexical retrieval of semantic knowledge and semantic control (but see Duffau et al., 2009; de Zubicaray et al., 2011; Harvey et al., 2013), reflecting the role of this pathway in connecting the IFG and the ATL (Catani et al., 2002). Indeed, XSL might engage both the left IFG and ATL. On the one hand, the IFG has been related to the retention of semantic short-term representations (Shivde and Thompson-Schill, 2004) and to the selection, activation, and inhibition of competing semantic alternatives (Thompson-Schill and Botvinick, 2006; Hoffman et al., 2009). Furthermore, it has been proposed that XSL relies on statistical learning mechanisms (Yu and Smith, 2007; Peñaloza et al., 2017), which have been associated with the IFG in both healthy (Karuza et al., 2013) and clinical populations (Peñaloza et al., 2015). On the other hand, the ATL plays a role in semantic processing (Bajada et al., 2016), object naming (Price et al., 2006), and semantic lexical retrieval (Schwartz et al., 2009), with WM in this region also being associated with the degradation of conceptual representations in semantic dementia (Agosta et al., 2010; Ralph et al., 2017). Moreover, the ATL is thought to operate as a hub that processes information about object-related associations (Patterson et al., 2007). Therefore, it is possible that the IFG is engaged during XSL through statistical learning mechanisms and that the ATL- 

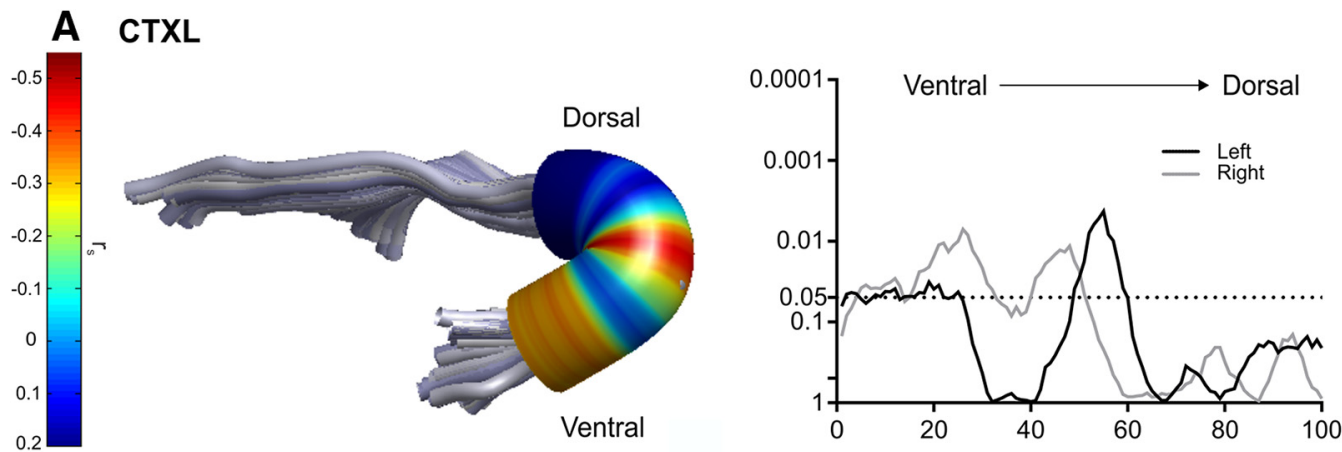

B XSL

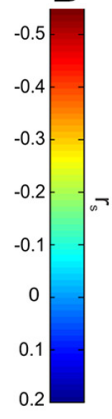

C

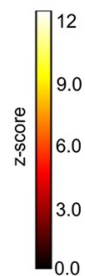

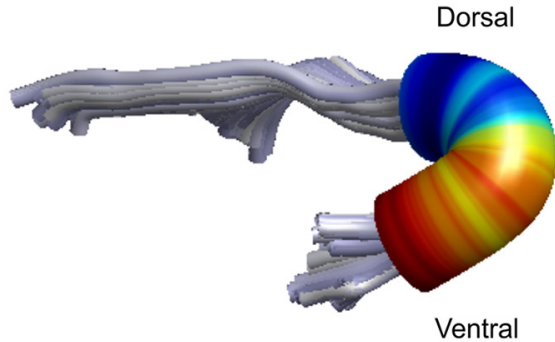

XSL ILF

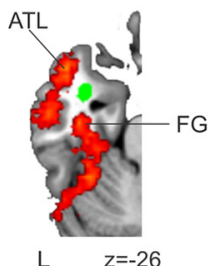

L $\quad z=-26$

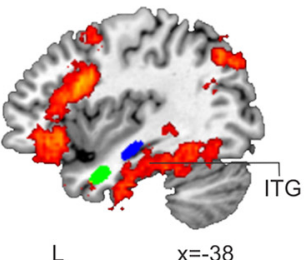

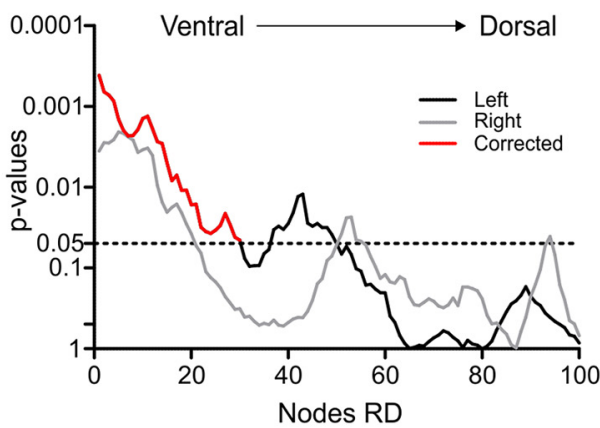

XSL UF
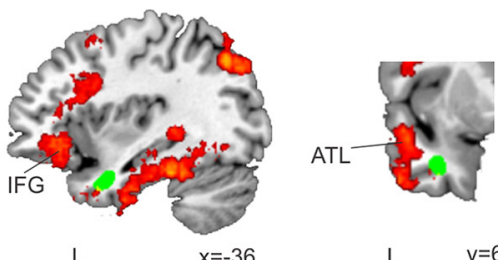

L $\quad y=6$

Figure 5. UF automatic dissections. Correlations between word-learning scores and RD values were calculated on a point-by-point basis using 100 nodes equidistantly placed along the UF. In $A$ and $\boldsymbol{B}$, a tract from an individual subject with a colored overlay representing the Spearman's rank-order $r_{s}$ value is shown on the left. The $p$ values associated with each $r_{s}$ value at each of the 100 points extracted with the automatic procedure are displayed in the right (left hemisphere in black; right hemisphere in gray; FWE-corrected clusters in red). $\boldsymbol{A}$, Results of the nonsignificant correlations between the WM microstructural properties of the left UF and individual success in CTXL are shown for the sake of completeness. $\boldsymbol{B}$, Results of the correlations between the WM microstructural properties of the left UF and individual success in XSL (in this case, FWE corrected for multiple comparisons). $\boldsymbol{C}$, Approximate location in MNI space of the result shown in $\boldsymbol{B}$ for the UF and in Figure $4 B$ for the ILF (see Materials and Methods), shown over a canonical T1 along a Neuro-Synth meta-analysis of semantic-related fMRI activity. Neurological convention is used, with MNI coordinates shown at the right bottom of each slice. In dark blue, portions of the ILF correlating with XSL (Fig. 4B). In green, portions of the UF correlating with XSL. L, Left hemisphere; ATL, anterior temporal lobe; ITG, inferior temporal gyrus; FG, fusiform gyrus.

CTXL

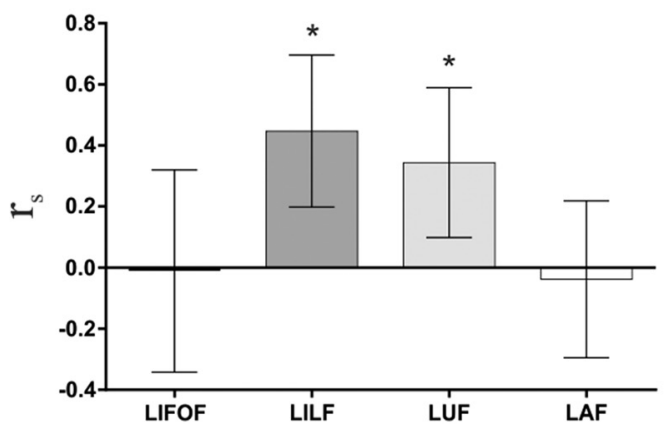

XSL

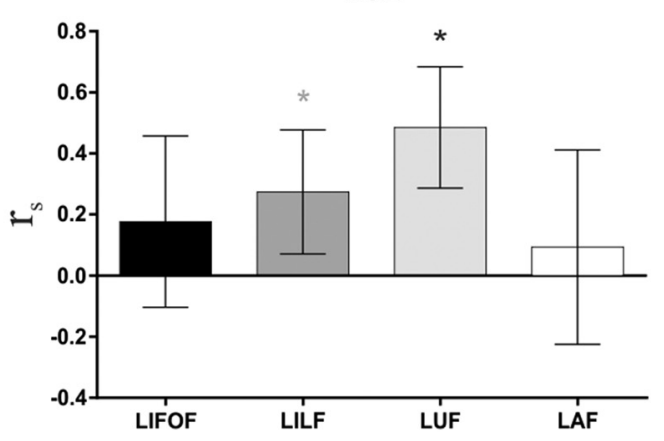

Figure 6. Results of the cross-validation approach. A cross-validation approach was used to assess whether the $100 \mathrm{RD}$ values from the left ILF and left UF actually predicted (instead of just correlated with) CTXL and XSL scores. Data for the left AF and left IFOF are also presented as a control. Bars represent average $r_{s}$ values ( \pm SD) denoting the goodness of fit for each tract as a predictor for each word-learning score. The $r_{s}$ value is computed using the predicted scores (calculated for 100 testing subsets of the data, using the coefficients obtained after fitting a model with their respective training sets; see Materials and Methods) and the real learning scores. The closer the $r_{s}$ value is to 1 , the more similar the predicted and real values are, and the better the prediction is. ${ }^{*} p<0.05 ; * p=0.0789$. 
receiving its contribution through the UF-integrates novel information about conceptual referents that have recently been disambiguated. We propose that the ILF, and especially the UF, may support the integration and retrieval of the correct representations for recently acquired words during XSL, although functional data are still needed to support this claim.

Research shows that the initial stage of word learning is a rapid process sustained by medial temporal lobe structures (Davis and Gaskell, 2009; Rodriguez-Fornells et al., 2009). Studies in both clinical (Gabrieli et al., 1988; Warren et al., 2016) and healthy populations (Breitenstein et al., 2005; Tuomiranta et al., 2014) implicate the hippocampus in new word learning, as well as when a CTXL paradigm is used (Mestres-Missé et al., 2010; Ripollés et al., 2016). In our study, the region in the anterior to mid-section of the ILF showing significant correlations with both CTXL and XSL is close to the hippocampus (Fig. 4C, light blue cluster). This could support the idea that initial information regarding word-to-meaning mappings reaches the medial temporal lobe via the ILF.

Remarkably, we found no significant correlations between semantic learning and the IFOF. In a previous DW-MRI wholebrain analysis (in standard space), we found a scattered pattern of results that showed significant correlations with CTXL not only for the ILF and UF, but also for the IFOF and even portions of the AF (Ripollés et al., 2014). This may suggest that fine-grained analyses (i.e., tractography) performed in native space can be more sensitive to subject-specific effects. There is still an ongoing debate regarding whether the IFOF or the ILF-UF route is the crucial pathway for semantic processing. While previous studies show that intraoperative electrostimulation of the IFOF- but not of the UF or ILF-induces disruptions in semantic processing (Duffau et al., 2005, 2009; Mandonnet et al., 2007), recent research in both healthy (Saur et al., 2008; Wong et al., 2011) and patient populations (Shinoura et al., 2010; Papagno et al., 2011; Harvey et al., 2013) shows that the ILF-UF pathway may also support semantic processing. Although we cannot rule out a role of the IFOF in semantic learning (note that the way in which we selected the ROIs might have constrained the IFOF temporal connections; Turken and Dronkers, 2011), our results lend support to the notion that although the IFOF might be sufficient during standard semantic processing, the ILF and UF may also support novel word-to-meaning mappings (Fig. 6). Remarkably, one can find a parallel between this indirect ILF-UF pathway and other indirect routes. For example, in the VTA-hippocampal loop (a circuit in the service of memory and learning), signals coming from the hippocampus must pass through several anatomical structures (e.g., ventral striatum) before reaching dopaminergic regions. This complexity is thought to be related to the need to control which information enters long-term memory (Lisman and Grace, 2005). In the same way, the ILF-UF pathway provides an indirect connection between temporal and inferior frontal regions that has the advantage of adding signals from the ATL.

In conclusion, we provide evidence for the involvement of the left ILF and UF in the acquisition of novel word-to-meaning mappings beyond their possible involvement in semantic processing. Current and previous (López-Barroso et al., 2013) findings are in line with views suggesting that the roles of the dorsal and ventral pathways in word learning resemble their roles in phonological and semantic processing, respectively. Conducting statistics along WM fiber bundles was found to be fundamental for a fine-grained identification of areas of specific importance for new word learning. We suggest that this approach can be generalized for the identification of WM bottlenecks in cognitive processing.

\section{References}

Agosta F, Henry RG, Migliaccio R, Neuhaus J, Miller BL, Dronkers NF, Brambati SM, Filippi M, Ogar JM, Wilson SM, Gorno-Tempini ML (2010) Language networks in semantic dementia. Brain 133:286-299. CrossRef Medline

Allport DA (1985) Distributed memory, modular subsystems and dysphasia. In: Current perspectives in dysphasia (Newman SK, Epstein R, eds), Edinburgh, UK: Churchill Livingstone.

Ashburner J (2007) A fast diffeomorphic image registration algorithm. Neuroimage 38:95-113. CrossRef Medline

Ashburner J, Friston KJ (2005) Unified segmentation. Neuroimage 26:839851. CrossRef Medline

Aung WY, Mar S, Benzinger TL (2013) Diffusion tensor MRI as a biomarker in axonal and myelin damage. Imaging Med 5:427-440. CrossRef Medline

Badre D, Wagner AD (2007) Left ventrolateral prefrontal cortex and the cognitive control of memory. Neuropsychologia 45:2883-2901. CrossRef Medline

Badre D, Poldrack RA, Paré-Blagoev EJ, Insler RZ, Wagner AD (2005) Dissociable controlled retrieval and generalized selection mechanisms in ventrolateral prefrontal cortex. Neuron 47:907-918. CrossRef Medline

Bajada CJ, Haroon HA, Azadbakht H, Parker GJ, Lambon Ralph MA, Cloutman LL (2016) The tract terminations in the temporal lobe: their location and associated functions. Cortex. Advance online publication. Retrieved October 12, 2017. doi:10.1016/j.cortex.2016.03.013. CrossRef Medline

Basser PJ, Pajevic S, Pierpaoli C, Duda J, Aldroubi A (2000) In vivo fiber tractography using DT-MRI data. Magn Reson Med 44:625-632. CrossRef Medline

Behrens TE, Johansen-Berg H (2005) Relating connectional architecture to grey matter function using diffusion imaging. Philos Trans R Soc Lond B Biol Sci 360:903-911. CrossRef Medline

Binder JR, Desai RH, Graves WW, Conant LL (2009) Where is the semantic system? A critical review and meta-analysis of 120 functional neuroimaging studies. Cereb Cortex 19:2767-2796. CrossRef Medline

Brainard DH (1997) The Psychophysics Toolbox. Spat Vis 10:433-436. CrossRef Medline

Breitenstein C, Jansen A, Deppe M, Foerster AF, Sommer J, Wolbers T, Knecht S (2005) Hippocampus activity differentiates good from poor learners of a novel lexicon. Neuroimage 25:958-968. CrossRef Medline

Brett M, Anton JL, Valabregue R, Poline JB (2002) Region of interest analysis using an SPM toolbox. Paper presented at 8th International Conference on Functional Mapping of the Human Brain, Sendai, Japan, June.

Catani M, Thiebaut de Schotten M (2008) A diffusion tensor imaging tractography atlas for virtual in vivo dissections. Cortex 44:1105-1132. CrossRef Medline

Catani M, Howard RJ, Pajevic S, Jones DK (2002) Virtual in vivo interactive dissection of white matter fasciculi in the human brain. Neuroimage 17: 77-94. CrossRef Medline

Catani M, Jones DK, Donato R, Ffytche DH (2003) Occipito-temporal connections in the human brain. Brain 126:2093-2107. CrossRef Medline

Catani M, Jones DK, ffytche DH (2005) Perisylvian language networks of the human brain. Ann Neurol 57:8-16. CrossRef Medline

Davis MH, Gaskell MG (2009) A complementary systems account of wordlearning: neural and behavioural evidence. Philos Trans R Soc Lond B Biol Sci 364:3773-3800. CrossRef Medline

de Zubicaray GI, Rose SE, McMahon KL (2011) The structure and connectivity of semantic memory in the healthy older adult brain. Neuroimage 54:1488-1494. CrossRef Medline

Dice LR (1945) Measures of the amount of ecologic association between species. Ecology 26:297-302. CrossRef

Dick AS, Tremblay P (2012) Beyond the arcuate fasciculus: consensus and controversy in the connectional anatomy of language. Brain 135:35293550. CrossRef Medline

Dodson CK, Travis KE, Ben-Shachar M, Feldman HM (2017) White matter microstructure of 6-year old children born preterm and full term. Neuroimage Clin 16:268-275. CrossRef Medline

Duffau H, Gatignol P, Mandonnet E, Peruzzi P, Tzourio-Mazoyer N, Capelle L (2005) New insights into the anatomo-functional connectivity of the 
semantic system: a study using cortico-subcortical electrostimulations. Brain 128:797-810. CrossRef Medline

Duffau H, Gatignol P, Moritz-Gasser S, Mandonnet E (2009) Is the left uncinate fasciculus essential for language? A cerebral stimulation study. J Neurol 256:382-389. CrossRef Medline

Dutoit P, Pagel N, Pierret f, Bataille O, van der Vreken O (1996) The MBROLA Project: towards a set of high-quality speech synthesizers free of use for non-commercial purposes. Paper presented at ICSLP 96: The Fourth International Conference on Spoken Language Processing. Philadelphia, October.

Fields RD (2008) White matter in learning, cognition and psychiatric disorders. Trends Neurosci 31:361-370. CrossRef Medline

Fiez JA (1997) Phonology, semantics, and the role of the left inferior prefrontal cortex. Hum Brain Mapp 5:79-83. CrossRef Medline

Forkel SJ, Thiebaut de Schotten M, Kawadler JM, Dell'Acqua F, Danek A, Catani M (2014) The anatomy of fronto-occipital connections from early blunt dissections to contemporary tractography. Cortex 56:73-84. CrossRef Medline

François C, Ripollés P, Bosch L, Garcia-Alix A, Muchart J, Sierpowska J, Fons C, Solé J, Rebollo M, Gaitán H, Rodriguez-Fornells A (2016) Language learning and brain reorganization in a 3.5-year-old child with left perinatal stroke revealed using structural and functional connectivity. Cortex 77:95-118. CrossRef Medline

Friston KJ, Ashburner J (2004) Generative and recognition models for neuroanatomy. Neuroimage 23:21-24. CrossRef Medline

Gabrieli JD, Cohen NJ, Corkin S (1988) The impaired learning of semantic knowledge following bilateral medial temporal-lobe resection. Brain Cogn 7:157-177. CrossRef Medline

Gow DW Jr (2012) The cortical organization of lexical knowledge: a dual lexicon model of spoken language processing. Brain Lang 121:273-288. CrossRef Medline

Gramfort A, Luessi M, Larson E, Engemann DA, Strohmeier D, Brodbeck C, Goj R, Jas M, Brooks T, Parkkonen L, Hämäläinen M (2013) MEG and EEG data analysis with MNE-Python. Front Neurosci 7:267. CrossRef Medline

Gramfort A, Luessi M, Larson E, Engemann DA, Strohmeier D, Brodbeck C, Parkkonen L, Hämäläinen MS (2014) MNE software for processing MEG and EEG data. Neuroimage 86:446-460. CrossRef Medline

Gupta P, Tisdale J (2009) Word-learning, phonological short-term memory, phonotactic probability and long-term memory: towards an integrated framework. Philos Trans R Soc Lond B Biol Sci 364:3755-3771. CrossRef Medline

Harvey DY, Wei T, Ellmore TM, Hamilton AC, Schnur TT (2013) Neuropsychological evidence for the functional role of the uncinate fasciculus in semantic control. Neuropsychologia 51:789-801. CrossRef Medline

Hickok G, Poeppel D (2007) The cortical organization of speech processing. Nat Rev Neurosci 8:393-402. CrossRef Medline

Hinrichs H, Scholz M, Tempelmann C, Woldorff MG, Dale AM, Heinze HJ (2000) Deconvolution of event-related fMRI responses in fast-rate experimental designs: tracking amplitude variations. J Cogn Neurosci 12 [Suppl 2]:76-89. CrossRef Medline

Hoffman P, Jefferies E, Ehsan S, Hopper S, Ralph MA (2009) Selective short-term memory deficits arise from impaired domain-general semantic control mechanisms. J Exp Psychol Learn Mem Cogn 35:137-156. CrossRef Medline

Hua K, Zhang J, Wakana S, Jiang H, Li X, Reich DS, Calabresi PA, Pekar JJ, van Zijl PC, Mori S (2008) Tract probability maps in stereotaxic spaces: analyses of white matter anatomy and tract-specific quantification. Neuroimage 39:336-347. CrossRef Medline

Jenkinson M, Beckmann CF, Behrens TE, Woolrich MW, Smith SM (2012) FSL. Neuroimage 62:782-790. CrossRef Medline

Kanai R, Rees G (2011) The structural basis of inter-individual differences in human behaviour and cognition. Nat Rev Neurosci 12:231-242. CrossRef Medline

Karuza EA, Newport EL, Aslin RN, Starling SJ, Tivarus ME, Bavelier D (2013) The neural correlates of statistical learning in a word segmentation task: an fMRI study. Brain Lang 127:46-54. CrossRef Medline

King JR, Pescetelli N, Dehaene S (2016) Brain mechanisms underlying the brief maintenance of seen and unseen sensory information. Neuron 92: 1122-1134. CrossRef Medline

Klawiter EC, Schmidt RE, Trinkaus K, Liang HF, Budde MD, Naismith RT, Song SK, Cross AH, Benzinger TL (2011) Radial diffusivity predicts de- myelination in ex vivo multiple sclerosis spinal cords. Neuroimage 55: 1454-1460. CrossRef Medline

Kronfeld-Duenias V, Amir O, Ezrati-Vinacour R, Civier O, Ben-Shachar M (2016) The frontal aslant tract underlies speech fluency in persistent developmental stuttering. Brain Struct Funct 221:365-381. CrossRef Medline

Lau EF, Phillips C, Poeppel D (2008) A cortical network for semantics: (de) constructing the N400. Nat Rev Neurosci 9:920-933. CrossRef Medline

Leemans A, Jones DK (2009) The B-matrix must be rotated when correcting for subject motion in DTI data. Magn Reson Med 61:1336-1349. CrossRef Medline

Lisman JE, Grace AA (2005) The hippocampal-VTA loop: controlling the entry of information into long-term memory. Neuron 46:703-713. CrossRef Medline

López-Barroso D, Catani M, Ripollés P, Dell'Acqua F, Rodríguez-Fornells A, de Diego-Balaguer R (2013) Word-learning is mediated by the left arcuate fasciculus. Proc Natl Acad Sci U S A 110:13168-13173. CrossRef Medline

Mandonnet E, Nouet A, Gatignol P, Capelle L, Duffau H (2007) Does the left inferior longitudinal fasciculus play a role in language? A brain stimulation study. Brain 130:623-629. CrossRef Medline

Medina TN, Snedeker J, Trueswell JC, Gleitman LR (2011) How words can and cannot be learned by observation. Proc Natl Acad Sci U S A 108: 9014-9019. CrossRef Medline

Mestres-Missé A, Càmara E, Rodriguez-Fornells A, Rotte M, Münte TF (2008) Functional neuroanatomy of meaning acquisition from context. J Cogn Neurosci 20:2153-2166. CrossRef Medline

Mestres-Missé A, Münte TF, Rodriguez-Fornells A (2009) Functional neuroanatomy of contextual acquisition of concrete and abstract words. J Cogn Neurosci 21:2154-2171. CrossRef Medline

Mestres-Missé A, Rodriguez-Fornells A, Münte TF (2010) Neural differences in the mapping of verb and noun concepts onto novel words. Neuroimage 49:2826-2835. CrossRef Medline

Mori S, Crain BJ, Chacko VP, van Zijl PC (1999) Three-dimensional tracking of axonal projections in the brain by magnetic resonance imaging. Ann Neurol 45:265-269. CrossRef Medline

Mummery CJ, Patterson K, Wise RJ, Vandenbergh R, Price CJ, Hodges JR (1999) Disrupted temporal lobe connections in semantic dementia. Brain 122:61-73. CrossRef Medline

Nation ISP (2001) Learning vocabulary in another language. Cambridge, UK: Cambridge UP.

Nichols TE, Holmes AP (2002) Nonparametric permutation tests for functional neuroimaging: a primer with examples. Hum Brain Mapp 15:1-25. CrossRef Medline

Papagno C, Miracapillo C, Casarotti A, Romero Lauro LJ, Castellano A, Falini A, Casaceli G, Fava E, Bello L (2011) What is the role of the uncinate fasciculus? Surgical removal and proper name retrieval. Brain 134:405414. CrossRef Medline

Patterson K, Nestor PJ, Rogers TT (2007) Where do you know what you know? The representation of semantic knowledge in the human brain. Nat Rev Neurosci 8:976-987. CrossRef Medline

Pedregosa F, Varoquaux G, Gramfort A, Michel V, Thirion B, Grisel O, Blondel M, Prettenhofer P, Weiss R, Dubourg V, Vanderplas K, Passos A, Cournapeau D, Brucher M, Perrot M, Duchesnay E (2011) Scikit-learn: machine learning in python. J Mach Learn Res 12:2825-2830.

Peñaloza C, Benetello A, Tuomiranta L, Heikius IM, Järvinen S, Majos MC, Cardona P, Juncadella M, Laine M, Martin N, Rodríguez-Fornells A (2015) Speech segmentation in aphasia. Aphasiology 29:724-743. CrossRef Medline

Peñaloza C, Mirman D, Cardona P, Juncadella M, Martin N, Laine M, Rodríguez-Fornells A (2017) Cross-situational word-learning in aphasia. Cortex 93:12-27. CrossRef Medline

Price CJ, McCrory E, Noppeney U, Mechelli A, Moore CJ, Biggio N, Devlin JT (2006) How reading differs from object naming at the neuronal level. Neuroimage 29:643-648. CrossRef Medline

Ralph MA, Jefferies E, Patterson K, Rogers TT (2017) The neural and computational bases of semantic cognition. Nat Rev Neurosci 18:42-55. CrossRef Medline

Rauschecker JP, Scott SK (2009) Maps and streams in the auditory cortex: nonhuman primates illuminate human speech processing. Nat Neurosci 12:718-724. CrossRef Medline

Reese TG, Heid O, Weisskoff RM, Wedeen VJ (2003) Reduction of eddycurrent-induced distortion in diffusion MRI using a twice-refocused spin echo. Magn Reson Med 49:177-182. CrossRef Medline 
Ripollés P, Marco-Pallarés J, de Diego-Balaguer R, Miró J, Falip M, Juncadella M, Rubio F, Rodriguez-Fornells A (2012) Analysis of automated methods for spatial normalization of lesioned brains. Neuroimage 60:12961306. CrossRef Medline

Ripollés P, Marco-Pallarés J, Hielscher U, Mestres-Missé A, Tempelmann C, Heinze HJ, Rodríguez-Fornells A, Noesselt T (2014) The role of reward in word-learning and its implications for language acquisition. Curr Biol 24:2606-2611. CrossRef Medline

Ripollés P, Marco-Pallares J, Alicart H, Tempelmann C, Rodriguez-Fornells A, Noesselt T (2016) Intrinsic monitoring of learning success facilitates memory encoding via the activation of the SN/VTA-hippocampal loop. Elife 5:e1744. CrossRef Medline

Rodríguez-Fornells A, Cunillera T, Mestres-Missé A, de Diego-Balaguer R (2009) Neurophysiological mechanisms involved in language learning in adults. Philos Trans R Soc Lond B Biol Sci 364:3711-3735. CrossRef Medline

Rojkova K, Volle E, Urbanski M, Humbert F, Dell'Acqua F, Thiebaut de Schotten M (2016) Atlasing the frontal lobe connections and their variability due to age and education: a spherical deconvolution tractography study. Brain Struct Funct 221:1751-1766. CrossRef Medline

Saur D, Kreher BW, Schnell S, Kümmerer D, Kellmeyer P, Vry MS, Umarova R, Musso M, Glauche V, Abel S, Huber W, Rijntjes M, Hennig J, Weiller C (2008) Ventral and dorsal pathways for language. Proc Natl Acad Sci U S A 105:18035-18040. CrossRef Medline

Schwartz MF, Kimberg DY, Walker GM, Faseyitan O, Brecher A, Dell GS, Coslett HB (2009) Anterior temporal involvement in semantic word retrieval: voxel-based lesion-symptom mapping evidence from aphasia. Brain 132:3411-3427. CrossRef Medline

Seghier ML, Ramlackhansingh A, Crinion J, Leff AP, Price CJ (2008) Lesion identification using unified segmentation-normalisation models and fuzzy clustering. Neuroimage 41:1253-1266. CrossRef Medline

Shinoura N, Suzuki Y, Tsukada M, Yoshida M, Yamada R, Tabei Y, Saito K, Koizumi T, Yagi K (2010) Deficits in the left inferior longitudinal fasciculus results in impairments in object naming. Neurocase 16:135-139. CrossRef Medline

Shivde G, Thompson-Schill SL (2004) Dissociating semantic and phonological maintenance using fMRI. Cogn Affect Behav Neurosci 4:10-19. CrossRef Medline

Smith LB, Suanda SH, Yu C (2014) The unrealized promise of infant statistical word-referent learning. Trends Cogn Sci 18:251-258. CrossRef Medline

Smith SM (2002) Fast robust automated brain extraction. Hum Brain Mapp 17:143-155. CrossRef Medline

Song SK, Sun SW, Ramsbottom MJ, Chang C, Russell J, Cross AH (2002) Dysmyelination revealed through MRI as increased radial (but unchanged axial) diffusion of water. Neuroimage 17:1429-1436. CrossRef Medline

Song SK, Yoshino J, Le TQ, Lin SJ, Sun SW, Cross AH, Armstrong RC (2005) Demyelination increases radial diffusivity in corpus callosum of mouse brain. Neuroimage 26:132-140. CrossRef Medline

Thiebaut de Schotten M, Ffytche DH, Bizzi A, Dell'Acqua F, Allin M, Walshe M, Murray R, Williams SC, Murphy DG, Catani M (2011a) Atlasing location, asymmetry and inter-subject variability of white matter tracts in the human brain with MR diffusion tractography. Neuroimage 54:49-59. CrossRef Medline

Thiebaut de Schotten M, Dell'Acqua F, Forkel SJ, Simmons A, Vergani F, Murphy DG, Catani M (2011b) A lateralized brain network for visuospatial attention. Nat Neurosci 14:1245-1246. CrossRef Medline
Thiebaut de Schotten M, Tomaiuolo F, Aiello M, Merola S, Silvetti M, Lecce F, Bartolomeo P, Doricchi F (2014) Damage to white matter pathways in subacute and chronic spatial neglect: a group study and 2 single-case studies with complete virtual "in vivo" tractography dissection. Cereb Cortex 24:691-706. CrossRef Medline

Thompson-Schill SL, Botvinick MM (2006) Resolving conflict: a response to Martin and Cheng (2006). Psychon Bull Rev 13:402-408. Medline

Travis KE, Ben-Shachar M, Myall NJ, Feldman HM (2016) Variations in the neurobiology of reading in children and adolescents born full term and preterm. Neuroimage Clin 11:555-565. CrossRef Medline

Trueswell JC, Medina TN, Hafri A, Gleitman LR (2013) Propose but verify: fast mapping meets cross-situational word-learning. Cogn Psychol 66: 126-156. CrossRef Medline

Tuomiranta LM, Càmara E, Froudist Walsh S, Ripollés P, Saunavaara JP, Parkkola R, Martin N, Rodríguez-Fornells A, Laine M (2014) Hidden word-learning capacity through orthography in aphasia. Cortex 50:174191. CrossRef Medline

Turken AU, Dronkers NF (2011) The neural architecture of the language comprehension network: converging evidence from lesion and connectivity analyses. Front Syst Neurosci 5:1. CrossRef Medline

van Daalen-Kapteijns M, Elshout-Mohr M, de Glopper K (2001) Deriving the meaning of unknown words from multiple contexts. Lang Learn 51: 145-181. CrossRef

Vaquero L, Rodríguez-Fornells A, Reiterer SM (2017) The left, the better: white-matter brain integrity predicts foreign language imitation ability. Cereb Cortex 27:3906-3917. CrossRef Medline

Vigneau M, Beaucousin V, Hervé PY, Duffau H, Crivello F, Houdé O, Mazoyer B, Tzourio-Mazoyer N (2006) Meta-analyzing left hemisphere language areas: phonology, semantics, and sentence processing. Neuroimage 30:1414-1432. CrossRef Medline

Wakana S, Caprihan A, Panzenboeck MM, Fallon JH, Perry M, Gollub RL, Hua K, Zhang J, Jiang H, Dubey P, Blitz A, van Zijl P, Mori S (2007) Reproducibility of quantitative tractography methods applied to cerebral white matter. Neuroimage 36:630-644. CrossRef Medline

Warren DE, Tranel D, Duff MC (2016) Impaired acquisition of new words after left temporal lobectomy despite normal fast-mapping behavior. Neuropsychologia 80:165-175. CrossRef Medline

Wilke M, de Haan B, Juenger H, Karnath HO (2011) Manual, semiautomated, and automated delineation of chronic brain lesions: a comparison of methods. Neuroimage 56:2038-2046. CrossRef Medline

Wong FC, Chandrasekaran B, Garibaldi K, Wong PC (2011) White matter anisotropy in the ventral language pathway predicts sound-to-wordlearning success. J Neurosci 31:8780-8785. CrossRef Medline

Yarkoni T, Poldrack RA, Nichols TE, Van Essen DC, Wager TD (2011) Large-scale automated synthesis of human functional neuroimaging data. Nat Methods 8:665-670. CrossRef Medline

Yeatman JD, Dougherty RF, Myall NJ, Wandell BA, Feldman HM (2012) Tract profiles of white matter properties: automating fiber-tract quantification. PLoS One 7:e49790. CrossRef Medline

Yu C, Smith LB (2007) Rapid word-learning under uncertainty via crosssituational statistics. Psychol Sci 18:414-420. CrossRef Medline

Zatorre RJ, Fields RD, Johansen-Berg H (2012) Plasticity in gray and white: neuroimaging changes in brain structure during learning. Nat Neurosci 15:528-536. CrossRef Medline

Zhao J, Thiebaut de Schotten M, Altarelli I, Dubois J, Ramus F (2016) Altered hemispheric lateralization of white matter pathways in developmental dyslexia: evidence from spherical deconvolution tractography. Cortex 76:51-62. CrossRef Medline 